\title{
Implementation and challenges of test, treat and track (T3) strategy for malaria case management in children under five years in the Bongo District, Ghana
}

\author{
Shiela Akanteele Agandaa ${ }^{1,2}$, Margaret Kweku ${ }^{1}$, Eric Agboli ${ }^{1}$, Mohamed Takase ${ }^{3}$, Wisdom Takramah ${ }^{1}$, Elvis Tarkang $^{4 *}$, and John Gyapong ${ }^{5}$ \\ ${ }^{1}$ Department of Epidemiology and Biostatistics, School of Public Health, University of Health and Allied Sciences, Ho, Ghana \\ ${ }^{2}$ Regional Hospital, Ghana Health Service, Bolgatanga \\ ${ }^{3}$ Evangelical Presbyterian University College, University in Ho, Ghana \\ ${ }^{4}$ Department of Population and Behavioural Sciences, School of Public Health, University of Health and Allied Sciences, Ho, Ghana \\ ${ }^{5}$ Office of the Vice Chancellor, University of Health and Allied Sciences, Ho, Ghana
}

\begin{abstract}
Background: Malaria is a very deadly vector borne disease, which causes high morbidity and mortality, especially among children less than five years. In 2010 , Ghana subscribed to the World Health Organization's recommendation and developed guidelines for implementing the test, treat and track (T3) strategy. This study examined the extent to which the T3 strategy was being adhered to by clinicians and the challenges with its implementation at various levels of the health system in the Bongo district of Ghana.
\end{abstract}

Method: This was a descriptive cross-sectional study carried out in March 2016 in 28 public health facilities comprising one (1) District Hospital, 6 Health Centres and 21 Community-based Health Planning and Services (CHPS) compounds. Semi-structured questionnaires were used to collect information from parents/ guardians of children under five years who visited the outpatient department (OPD). Information collected included whether the children reported with fever, were tested for malaria, treated with an antimalarial and asked to return to the health facility for review. The clinicians were asked if they were trained on the T3 strategy and the challenges they had with the implementation.

Results: Overall, 322 (91.2\%) out of the 353 children with fever were tested and 318 (98.8\%) of them were positive for malaria parasites. All (353) children including those $31(8.8 \%)$ not tested were treated with antimalarial drugs. Majority $351(99.4 \%)$ of the children were treated with ACTs and only $2(0.6 \%)$ were treated with Quinine. Less than half $167(48.3 \%)$ were asked to return for review. Only 150(42.5\%) of the children completed the T3 protocol. Completing T3 at facility level was highest at the CHPS compound 72 (60.5\%), followed by the District Hospital 32 (39.5\%) and the least was the Health Centre 46 (30.1\%). Completing T3 by clinicians was highest among Doctors 4 (80.0\%), followed by Health Assistants 24 (51.1\%), Nurses 92 (47.2\%) and the least was Medical/Physician Assistants (MA/ PA) 30(28.3\%). Overall, the main challenges confronting clinicians were frequent malaria rapid diagnostic test (RDT) kits stock-outs 11(39.3\%), lack of diagnostic facilities 10 (35.7\%), ACTs stock-outs 1 (3.6\%) and RDTs not giving accurate or valid results 3 (10.7\%). These challenges were mainly with clinicians at the CHPS compounds and the health centres. The District hospital had no challenges with logistics.

Conclusion: Testing fever cases for malaria parasitaemia was very high and ACTs were the main drugs for treating malaria at all levels. The main determinant of completing T3 was asking clients to return for review. Compliance with the T3 strategy was better at the CHPS compounds than Hospitals and Health Centres. Doctors and Health Assistants were more likely to complete T3 than nurses and MA/PA.

Recommendations: The National Malaria Control Programme (NMCP) should ensure that CHPS compounds and Health Centres are regularly stocked with RDTs and ACTs. More training and monitoring support for clinicians are required to improve patient tracking and management of non-malaria fever cases at the lower levels of the health system.

\section{Abbreviations}

ACT: Artemisinin-Based Combination Therapy; CHPS: Community-based Health Planning and Services; DHIMS: District Health Information Management system; GHS: Ghana Health Service; NMCP: National Malaria Control Program; OPD: Outpatient Department; RDT: Rapid Diagnostic Test; SP: Sulfadoxinepyramethamine; SPSS: Statistical Package for Social Sciences; T3: Test Treat and Track; WHO: World Health Organization

\section{Background}

Malaria is a very deadly vector-borne disease, which causes high morbidity and mortality, especially among children under five years according to World Health Organization (WHO) [1]. In 2014, 198 million cases of malaria and 584,000 deaths worldwide were recorded and most of the deaths due to malaria occurred in Africa and mostly in

Correspondence to: Elvis Tarkang, Department of Population and Behavioural Sciences, School of Public Health, University of Health and Allied Sciences, Ho, Ghana, Tel: +233 505754478; E-mail: ebeyang1@yahoo.com

Key words: T3 strategy, malaria, health facility level, clinicians, children under five years, bongo district

Received: September 21, 2016; Accepted: October 20, 2016; Published: October 24, 2016 
Aganda S, et al. (2016) Implementation and challenges of test, treat and track (T3) strategy for malaria case management in children under five years in the Bongo District, Ghana

\section{children (78\%) [1].}

Studies have shown that majority of the public primary health care facilities lacked diagnostic facilities including microscopes. In situations where testing facilities were available, personnel to cope with the high volumes of work were inadequate. Due to these, fever in the past in many endemic countries was equated to malaria and was diagnosed as malaria and treated presumptively [2]. It was reported that, clinicians' adherence to the negative malaria rapid diagnostic test (RDT) result was very poor in all age groups and the common concern among health staff is that negative tests do not definitively rule out malaria [3-5]. It is evident that rapid diagnostic test for malaria provides an opportunity for improving care, diagnosis and better disease management $[6,7]$.

Drugs for treating malaria have changed for the past decade. Artemisinin-based combination therapy (ACT) has been recommended by WHO for use as the first line treatment for uncomplicated malaria in malaria endemic countries and Quinine or injectable artesunate are recommended for used in severe malaria. There has been a massive reduction in malaria related morbidity and mortality in regions of high endemicity in the last decade, partly due to the effective use of ACT regimens [8]. Also, the utilization of ACTs as a first line therapy for uncomplicated malaria has significantly improved [9].

Reports have shown that in Ghana and Kenya, the probability of fever that could be attributed to malaria was as high as $61 \%$ and $67 \%$ respectively [10]. It has also been reported that in Kenya, less than $40 \%$ of febrile children under five years were tested [11] and in Ghana 73\% of children were presumptively diagnosed and treated for malaria [12].

WHO therefore, recommended another malaria control measure based on the T3 (Test, Treat and Track) strategy [13]. That is, every suspected case of malaria must be confirmed by microscopy or a rapid diagnostic test (RDT) before treatment is given with antimalarial drugs. This is to help reduce the emergence and spread of drug resistance by reserving antimalarial drugs for those who actually have the disease. To ensure effective control, every malaria case should be tracked in a surveillance system [13]. Ability to Test, Treat and Track will enable malaria elimination to be seriously anticipated [14].

In 2013, Ghana subscribed to the WHO's recommendation and developed guidelines for the implementation of the test, treat and track (T3) strategy by updating the 2009 malaria case management guidelines [15-17]. Health care providers at various levels of the health system received training on the T3 strategy for the new malaria case management.

In Ghana, compliance with the guidelines on ACT use is low (66.7\%) [18]. The cost-effectiveness of implementing test-based management of malaria hinges on health workers adhering to test results and restricting ACTs to test-positive cases while looking for other causes of fever in the test-negative cases [10].

In Bongo District, according to the District Health Information Management Systems (DHIMS), malaria is number one among the top ten OPD cases which accounted for 67,128 (43.6\%), 73,829 (39.7\%) $30,110(21.5 \%)$ in 2012, 2013 and 2014 respectively. Report also indicates that malaria cases tested were $13,272(19.8 \%)$ in $2012,22,186$ $(30.1 \%)$ in 2013 , and $18,407(60.1 \%)$ in 2014 . Malaria cases tested among children under five years were 6,375 (23.7\%) in 2012, 9,214 (32.1\%) in 2013 and 6,766 (63.5\%) in 2014 [19].

This study assessed compliance with the implementation of the T3 strategy among children under five years in the Bongo district. It also examined some challenges confronting the implementation of the $\mathrm{T} 3$ strategy.

\section{Materials and method}

\section{Study area}

Bongo district is one of the 13 administrative districts in the Upper East Region. The district which was carved out of Bolgatanga in 1988, shares boundaries with Burkina Faso to the East, Kassena-Nankana to the North, Kassena-Nankana to the West and Bolgatanga district to the South. The district has a total population of 89,741 people [20].

Bongo Township serves as the district capital. It is one of the most densely populated districts in the country with 187 inhabitants per square kilometre and a land area of 459.5 square kilometres. The district has a total of 134 communities scattered in small-dispersed settlements. The district is divided into six sub-districts. It has 28 health facilities, which include District Hospital (1), Health Centres (6) and CHPS compounds (21). There are also 5 Nutrition feeding Centres and 69 Expanded Programme on Immunisation (EPI) Outreach points. Farming is the main occupation, followed by a few trading activities in the economic sector [20]. Bongo district has one big dam and several satellite dams, lots of streams and rivers as well as cracked rocky surfaces that collect fresh water for breeding of mosquitoes. Malaria transmission is all year round but peaks in the rainy seasons in April to June.

Bongo district has benefited from several malaria interventions including Indoor Residual Spraying, Malaria Case Management with effective drugs and the use of Long Lasting Insecticide Nets (LLIN) [19]. All health facilities in the district collect RDTs (CareStart ${ }^{\mathrm{TM}}$ Malaria HRP2 test kit) (Access Bio Inc, New Jersey, USA) from the District Health Directorate for the rapid qualitative detection of Malaria Histidine-rich Protein 2 (HRP2) in human blood as an aid in the diagnosis of malaria infection.

\section{Study design}

A descriptive cross-sectional study was carried out in March 2016. Data was collected from participants using a semi-structured questionnaire. Information was collected from parents/guardians who brought their children under five years old to the OPD. Information collected included whether the children were tested before treatment was given, treatment was given in accordance with the treatment guideline and whether the children were asked to return for followup. Clinicians at the health facilities were also interviewed. Information collected included whether they received training on the T3 strategy, and the challenges confronting them in the implementation of the T3 strategy.

\section{Study population}

The study involved children less than five years who visited the health facilities with fever. It also involved clinicians at various health facilities.

\section{Sample size determination and sampling}

Sample size: Using the Cochran W.C formula for samples calculation stated below:

$$
n=\frac{\left(\mathrm{Z} \frac{\alpha}{2}\right)^{2} p(1-p)}{(e)^{2}}
$$

Where: $n$ is the expected sample size, $z(\mathbf{1 . 9 6 )}$ is $\mathrm{z}$ statistics for a 
Aganda S, et al. (2016) Implementation and challenges of test, treat and track (T3) strategy for malaria case management in children under five years in the Bongo District, Ghana

confidence level of $95 \%, \boldsymbol{e}(5 \%)$ is the margin of error and $\boldsymbol{p}(32 \%)$ the estimated proportion of malaria in children under five in Bongo [19]. The calculation is as follows:

$$
n=\frac{1.96^{2} \times 0.32(1-0.32)}{0.05^{2}}=334.23
$$

Adding $5 \%$ of 334 children to compensate for errors that might occur during data collection, a total sample size of 351 children under five were needed. A total of 353 children under five were recruited for the study. All twenty-eight (28) facilities were included in the study and therefore a total of 28 clinicians (one from each facility) were interviewed.

Sampling: All the twenty-eight (28) facilities were used for the study. This included one district hospital, 6 health centres and 21CHPS compounds. A probability (simple random) sampling technique was used for the selection of respondents in each facility. The lottery method was used and it was the non-replacement type. In doing that, the expected number of respondents for each facility was estimated. This was done by counting the number of children under five with malaria that reported at the facility in the same period of data collection of the previous year (2015) for each facility. The assumption was that the same number of cases (sample frame or potential respondents) would be seen at the facility. Once the sampling frame for each facility was obtained and the number of respondents to be interviewed for each facility was known, 'yes' and 'no' was written on pieces of papers and folded.

The number of 'yes' was equal to the number of respondents per facility and the number of 'no' was equal to the number of cases remaining after subtracting the respondents from the total number of cases for each facility. All those who were eligible, randomly picked one paper. Those who picked 'yes' were interviewed and those who picked 'no' were not interviewed. In a situation where the number of malaria cases were less than or equal to the number of respondents to be interviewed for each facility, then all those who visited the facility and were within the study population, were interviewed until the number of respondents for that facility was obtained. The respondents were proportionately distributed to the facilities based on 2015 malaria cases in children under-five years.

\section{Data collection}

Pre-tested, semi-structured questionnaires were used to collect the data. The questionnaire was administered to parents/guardians of eligible children below five years immediately after they had received treatment and were exiting the health facility. The exit interview was conducted in a convenient and conducive place, in English language, and in Gurune for respondents who did not understand or speak English.

Also, twenty-eight (28) clinicians were interviewed using an interview guide on factors associated with compliance to the implementation of the T3 strategy. One clinician was interviewed in each facility. In an instance where in a facility the clinicians were more than one, simple random sampling was used to select one.

\section{Statistical analysis}

Data from respondents were entered twice into a database using EPI data software. The data were checked and validated using customized validation programmes. The cleaned data were analysed using Statistical Package for Social Sciences (SPSS) version 20. Results were displayed in tables and graphs according to the variables studied. Chi-square test was used to determine association between each demographic categorical variable and dependent variables. A bivariate correlation was performed by feeding test, treat and track variables into compliance with T3 to determine which of the variables were the key determinant of completion of T3. Chi-square test result with p-value less than 0.05 was considered significant.

\section{Ethical issues}

Before commencement of the study, ethical clearance was obtained from the Ghana Health Service Ethical Review Committee (ID NO: GHS-ERC-12/02/16). Permission was also sought from the District Management Team and the Hospital management. An informed consent was obtained from the respondents before the questionnaire was administered. Participants were assured of confidentiality and privacy throughout the study. All information concerning individual subjects remained anonymous and confidential.

\section{Results}

Table 1 shows that out of a total of 353 children enrolled 119 (33.7\%) were from the CHPS compounds, 153 (43.3\%) from the Health Centres and 81 (23.0\%) from the District Hospital. The mean age of the children was $25.8 \pm 16.8$ months. A greater proportion $193(54.7 \%)$ of the children were males while 160 (45.3\%) were females.

Table 2 shows that out of the 28 clinicians interviewed, 20 (71.4\%) were from the CHPS compounds, 6 (21.4\%) from the Health Centres and 2 (7.2\%) from the Hospital. Majority, 15(53.6\%) of the clinicians were nurses followed by Health Assistants 11 (39.3\%), MA/PA 1 (3.6\%) and Doctor 1 (3.6\%). Most of the clinicians, 21 (75.0\%) were aware of T3, $22(78.6 \%)$ received training on case management and all received training on RDT use. More than half, 195(55.2\%) of the children were seen by Nurses, 106 (30.0\%) by Medical/physician assistants, 47 (13.3\%) by Health Assistants and only $5(1.4 \%)$ were attended to by a Doctor.

Table 3 shows that, overall, 322 (91.2\%) out of the 353 children had their blood tested while $31(8.8 \%)$ did not. The blood test done was high at the hospital level, (97.5\%) followed by health centres, $(89.5 \%)$ and the lowest was at the CHPS compounds, (89.1\%). Majority, (98.8\%) of the 322 children tested for malaria were positive. There was no statistically significant difference between blood test done and no test done when compared with the level of health facility $\left(x^{2}=5.2 ; \mathrm{P}=0.073\right)$. Similarly, the difference in test outcome (malaria parasites present or absent)

Table 1. Background characteristics of children under five years by level of health facility.

\begin{tabular}{|c|c|c|c|c|c|c|}
\hline \multicolumn{7}{|c|}{ Level of health facility } \\
\hline Characteristic & $\begin{array}{l}\text { CHPS } \\
\text { compound } \\
\text { n }(\%)\end{array}$ & $\begin{array}{l}\text { Health } \\
\text { Centre } \\
\text { n (\%) }\end{array}$ & $\begin{array}{l}\text { District } \\
\text { Hospital } \\
\text { n (\%) }\end{array}$ & $\begin{array}{c}\text { Total } \\
\text { N (\%) }\end{array}$ & $\begin{array}{l}\text { Pearson } \\
\text { Chi-Square } \\
\left(x^{2}\right)\end{array}$ & p-value \\
\hline $\begin{array}{l}\text { Number } \\
\text { enrolled }\end{array}$ & $119(33.7)$ & $153(43.3)$ & $81(23.0)$ & $353(100)$ & & \\
\hline $\begin{array}{l}\text { Mean age } \\
\text { (SD) }\end{array}$ & $26.6(17.4)$ & $28.3(16.5)$ & $19.9(15.1)$ & $25.8(16.8)$ & & \\
\hline \multicolumn{7}{|c|}{ Age groups (in months) } \\
\hline $0-11$ & $31(8.8)$ & $23(6.5)$ & $27(7.6)$ & $81(22.9)$ & & \\
\hline $12-23$ & $21(5.9)$ & $29(8.2)$ & $24(6.9)$ & $74(21.0)$ & & \\
\hline $24-35$ & $17(4.8)$ & $31(8.8)$ & $9(2.5)$ & $57(16.1)$ & 21.6 & 0.006 \\
\hline $36-47$ & $19(5.4)$ & $23(6.5)$ & $9(2.5)$ & $51(14.4)$ & & \\
\hline $48-59$ & $31(8.8)$ & $47(13.3)$ & $12(3.4)$ & $90(25.5)$ & & \\
\hline \multicolumn{7}{|l|}{ Sex } \\
\hline Male & $56(15.9)$ & $75(21.2)$ & $62(17.6)$ & $193(54.7)$ & & \\
\hline Female & $63(17.8)$ & $78(22.1)$ & $19(5.4)$ & $160(45.3)$ & 20.4 & 0.000 \\
\hline
\end{tabular}


Aganda S, et al. (2016) Implementation and challenges of test, treat and track (T3) strategy for malaria case management in children under five years in the Bongo District, Ghana

between health facilities was not statistically significant $\left(x^{2}=5.5 ; \mathrm{p}\right.$ 0.065 ). The main reasons given by the clinicians for not testing were RDT stock-out (87.1\%), and laboratory not available to test (3.3\%). The rest of the respondents did not give any reason (Figure 1).

\section{Treatment with antimalarial drugs}

Almost all 351 (99.4\%) children were treated for malaria using ACTs except $2(0.6 \%)$ who were treated with Quinine, which is used as second line drug, or for severe malaria. Majority, 320 (91.2\%) were treated with ACTs based on test results, while $31(8.8 \%)$ were treated without testing (Table 4).

\section{Tracking of patients}

One hundred and eighty six (52.7\%) children were not tracked. Out of $167(47.3 \%)$ who were asked to return for review, 77 (46.1\%) were asked to return on specific days while 90 (53.9\%) were asked to return only if the child's condition did not improve. Majority, (70.6\%) of the children seen at the CHPS compound, were asked to return for review, while only $39.5 \%$ at the Hospital and $33.3 \%$ at the Health Centres were asked to return for review (Table 4).

Table 4 shows that majority, 4 (80.0\%) out of 5 children attended to by doctors were asked to return for review followed by the nurses, 109 (55.9\%) out of 195, then Health Assistants, 24 (51.1\%) out of 47 and the

Table 2. Background characteristics of clinicians interviewed.

\begin{tabular}{|c|c|c|c|c|}
\hline \multicolumn{5}{|l|}{ Level of health facility } \\
\hline Characteristic & $\begin{array}{c}\text { CHPS } \\
\text { Compound } \\
\text { n (\%) }\end{array}$ & $\begin{array}{l}\text { Health } \\
\text { Centre } \\
\text { n }(\%)\end{array}$ & $\begin{array}{c}\text { District } \\
\text { hospital } \\
\text { n (\%) }\end{array}$ & $\begin{array}{l}\text { Total } \\
\text { n (\%) }\end{array}$ \\
\hline Number of clinicians & 20 & 6 & 2 & $28(100 \%)$ \\
\hline \multicolumn{5}{|l|}{ Category of clinicians } \\
\hline Health Assistant & $9(45.0)$ & $2(33.3)$ & $0(0.0)$ & $11(39.3)$ \\
\hline Nurse & $11(55.0)$ & $3(50)$ & $1(3.6)$ & $15(53.6)$ \\
\hline Medical/physician assistant & $0(0.0)$ & $1(16.7)$ & $0(0.0)$ & $1(3.6)$ \\
\hline Doctor & $0(0.00)$ & $0(0.00)$ & $1(3.6)$ & $1(3.6)$ \\
\hline $\begin{array}{l}\text { Number of clinicians } \\
\text { aware of T3 }\end{array}$ & $15(75)$ & $4(66.7)$ & $2(100)$ & $21(75.0)$ \\
\hline $\begin{array}{l}\text { Clinicians who received } \\
\text { training on case } \\
\text { management }\end{array}$ & $16(80.0)$ & $4(66.7)$ & $2(100)$ & $22(78.6)$ \\
\hline $\begin{array}{l}\text { Clinicians who received } \\
\text { training on RDT }\end{array}$ & $20(100)$ & $6(100)$ & $2(100)$ & $28(100)$ \\
\hline \multicolumn{5}{|c|}{ Children attended to by each category of clinicians } \\
\hline Children seen at each level & 119 & 153 & 81 & $353(100)$ \\
\hline Health Assistant & $35(29.4)$ & $12(7.8)$ & $0(0.00)$ & $47(13.3)$ \\
\hline Nurse & $83(69.7)$ & $97(63.4)$ & $13(16.0)$ & $195(55.2)$ \\
\hline Medical/physician assistant & $1(0.8)$ & $42(27.5)$ & $63(77.8)$ & $106(30.0)$ \\
\hline Doctor & $0(0.0)$ & $0(0.0)$ & $5(6.2)$ & $5(1.4)$ \\
\hline
\end{tabular}

Table 3. Distribution of blood test and test outcome by levels of health facility. least was medical/Physician assistants, 30 (28.3\%) out of 106 patients they attended to.

Table 5 shows that compliance with T3 followed the same pattern with review as it gave a positive correlation co-efficient of almost 1 with $\mathrm{p}<0.001$. On the other hand, both Blood test and Treatment did not show any strong pattern as their correlation co-efficients were near zero with p-values of $<0.001$ and 0.224 respectively.

\section{Complete T3 strategy adherence}

Overall, only $42.5 \%$ out of the 353 children completed the T3 protocol by being tested, treated and asked to return for review. Majority, (57.5\%) of the children, missed a "T" and thus did not complete the protocol (Table 6).

More than half, $(60.5 \%)$ of children who were seen at the CHPS compounds, completed the T3, while only $39.5 \%$ and $30.1 \%$ of those seen at the District Hospital and Health Centres respectively, completed the T3. Majority, 4 out of $5(80.0 \%)$ of the children attended to by a doctor completed the T3 (tested, treated and asked to come for review). Half (51.1\%) of those attended to by Health Assistants, $47.2 \%$ by Nurses and only $28.3 \%$ of those seen by MA/PA completed the T3 (Table 6).

\section{Challenges to the implementation of T3}

A total of 28 clinicians were interviewed on challenges they faced with the implementation of the T3 strategy. Out of the 28 clinicians interviewed, 11 (39.3\%) mentioned frequent RDT stock-outs as the major challenge followed by lack of diagnostic facilities, $10(35.7 \%)$ with the least being frequent ACTs stock-outs 1 (3.6\%) (Figure 2). The main challenge at the CHPS compounds was frequent RDT stock-outs, whilst lack of diagnostic facilities was a major challenge at the Health Centre level. The District Hospital however, did not have any challenge (Figure 3).

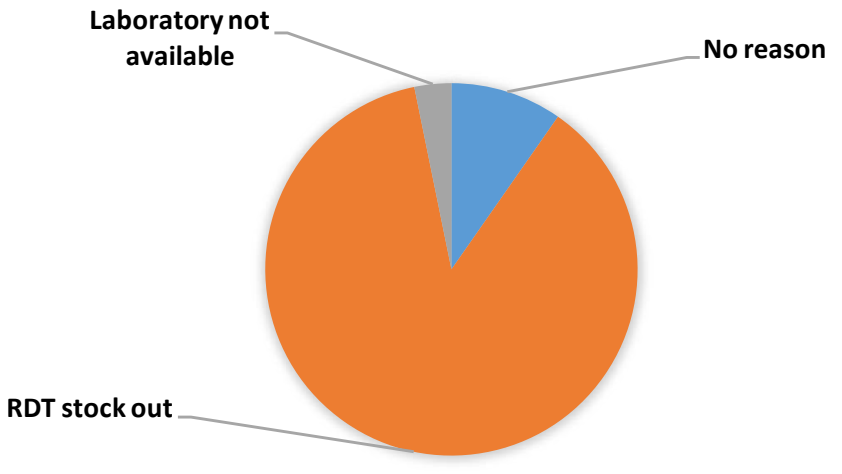

Figure 1. Reasons given for not testing the children

\begin{tabular}{|l|c|c|c|c|c|}
\hline Level of health facility & $\begin{array}{c}\text { CHPS compound } \\
\mathbf{n \%}\end{array}$ & $\begin{array}{c}\text { Health Centre } \\
\text { n (\%) }\end{array}$ & $\begin{array}{c}\text { District hospital } \\
\text { n (\%) }\end{array}$ & $\begin{array}{c}\text { Total } \\
\text { N (\%) }\end{array}$ \\
\hline Test & $119(33.7)$ & $153(43.3)$ & $81(23.0)$ & $353(100)$ \\
\hline Number enrolled & $13(10.9 \%)$ & $16(10.5)$ & $2(2.5)$ & $31(8.8)$ \\
\hline No blood test done & $106(89.1)$ & $137(89.5)$ & $79(97.5)$ & $322(91.2)$ \\
\hline Blood test done & \multicolumn{3}{|c|}{ Pearson } & \\
\hline Test outcome & 106 & 137 & 79 & $322(100)$ \\
\hline Number enrolled & $106(100)$ & $133(97.1)$ & $79(100)$ & $318(98.8)$ \\
\hline $\begin{array}{l}\text { Laboratory confirmed malaria } \\
\text { positive }\end{array}$ & $0(0.0)$ & $4(2.9)$ & 0.073 & \\
\hline Unknown & & & \\
\hline
\end{tabular}


Aganda S, et al. (2016) Implementation and challenges of test, treat and track (T3) strategy for malaria case management in children under five years in the Bongo District, Ghana

Table 4. Proportion of children who complied with $\mathrm{T} 3$ strategy at various health facility levels.

\begin{tabular}{|c|c|c|c|}
\hline & $\begin{array}{c}\text { Tested } \\
\text { Number (\%) }\end{array}$ & $\begin{array}{c}\text { Not tested } \\
\text { Number (\%) }\end{array}$ & Total \\
\hline \multicolumn{4}{|l|}{ Tested by Level of health facility } \\
\hline CHPS compound & $105(91.4)$ & $10(8.6)$ & 115 \\
\hline Health Centre & 133(90.5) & $14(9.5)$ & 147 \\
\hline District hospital & $79(98.7)$ & $1(1.3)$ & 80 \\
\hline Total & 317 & 25 & 342 \\
\hline \multicolumn{4}{|l|}{ Treatment Received } \\
\hline & Treatment with ACT & Not treated with ACT & \\
\hline \multirow[t]{2}{*}{ Treated with antimalarial drugs } & $351(99.4)$ & $2(0.6)$ & 353 \\
\hline & Tested and treated with ACTs & Not tested but treatment with ACTs & \\
\hline Treated with ACTs based on test results & $320(91.2)$ & $31(8.8)$ & $351(100)$ \\
\hline \multicolumn{4}{|c|}{ Children asked to return for review by level of facility } \\
\hline & Return for review & No review & \\
\hline CHPS compound & $84(70.6)$ & $35(29.4)$ & 119 \\
\hline Health Centre & $51(33.3)$ & $102(66.7)$ & 153 \\
\hline District hospital & $32(39.5)$ & $49(60.5)$ & 81 \\
\hline Total & $167(47.3)$ & $186(52.7)$ & 353 \\
\hline \multicolumn{4}{|c|}{ Category of clinicians who asked children to return for review by level of facility } \\
\hline & Return for review & No review & \\
\hline Health Assistant & $24(51.1)$ & 23(48.9) & 47 \\
\hline Nurse & $109(55.9)$ & $86(44.1)$ & 195 \\
\hline Medical/Physician assistant & $30(28.3)$ & $76(71.7)$ & 106 \\
\hline Doctor & $4(80.0)$ & $1(20.0)$ & 5 \\
\hline Total & $167(47.3)$ & $186(52.7)$ & 353 \\
\hline
\end{tabular}

Table 5. Bivariate correlation of Testing, treatment with ACT, Review with Compliance to $\mathrm{T} 3$.

\begin{tabular}{|l|c|c|}
\hline Variable & Correlation co-efficient & p-value \\
\hline Blood test & 0.27 & $<0.001$ \\
\hline Treatment with ACT & 0.07 & 0.224 \\
\hline Return for Review & 0.91 & $<0.001$ \\
\hline
\end{tabular}

Table 6. Proportion of children who completed T3 Protocol.

\begin{tabular}{|l|c|c|c|}
\hline \multicolumn{1}{|c|}{$\begin{array}{c}\text { Completed T3 } \\
\text { protocol N (\%) }\end{array}$} & $\begin{array}{c}\text { Did not complete } \\
\text { T3 N (\%) }\end{array}$ & Total \\
\hline Completion by Level of health facility & \multicolumn{1}{|c|}{} \\
\hline CHPS compound & $72(60.5)$ & $47(39.5)$ & 119 \\
\hline Health Centre & $46(30.1)$ & $107(69.9)$ & 153 \\
\hline District hospital & $32(39.5)$ & $49(60.5)$ & 81 \\
\hline Total & $150(42.5)$ & $203(57.5)$ & 352 \\
\hline Category of clinicians who attended to child & & \\
\hline Health Assistant & $24(51.1)$ & $23(48.9)$ & 47 \\
\hline Nurse & $92(47.2)$ & $103(52.8)$ & 195 \\
\hline $\begin{array}{l}\text { Medical/Physician } \\
\text { assistant }\end{array}$ & $30(28.3)$ & $76(71.7)$ & 106 \\
\hline Doctor & $4(80.0)$ & $1(20.0)$ & 5 \\
\hline
\end{tabular}

\section{Discussion}

The WHO recommends that every suspected malaria case should be tested, in other words malaria should be diagnosed either by microscopy or malaria RDT in all suspected malaria cases before treatment is administered. WHO also recommends ACTs as the firstline treatment for uncomplicated malaria caused by plasmodium falciparum, and above all, malaria cases should be tracked in a surveillance system following introduction of the T3 strategy in 2010 [21-23].

Rapid diagnostic test for malaria provides an opportunity for improved point of care diagnosis and better disease management

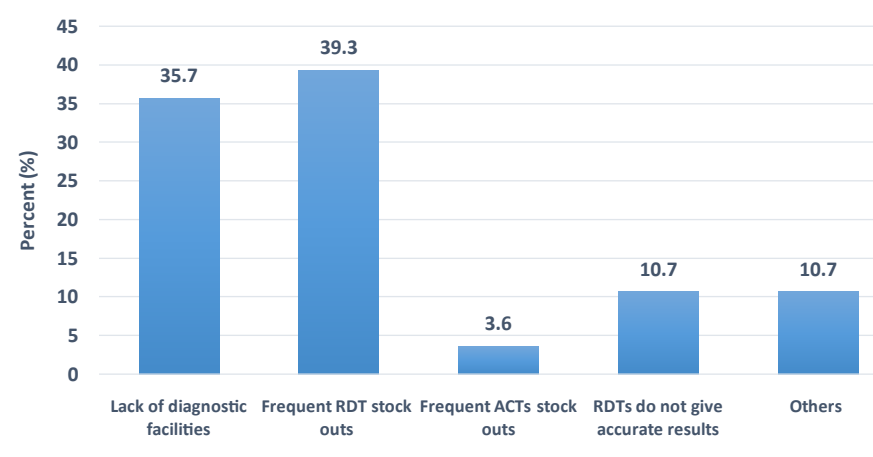

Figure 2. Challenges to the implementation of $\mathrm{T} 3$ strategy at various health facilities. a CHPS Compound $\quad$ Health Centre District Hospital

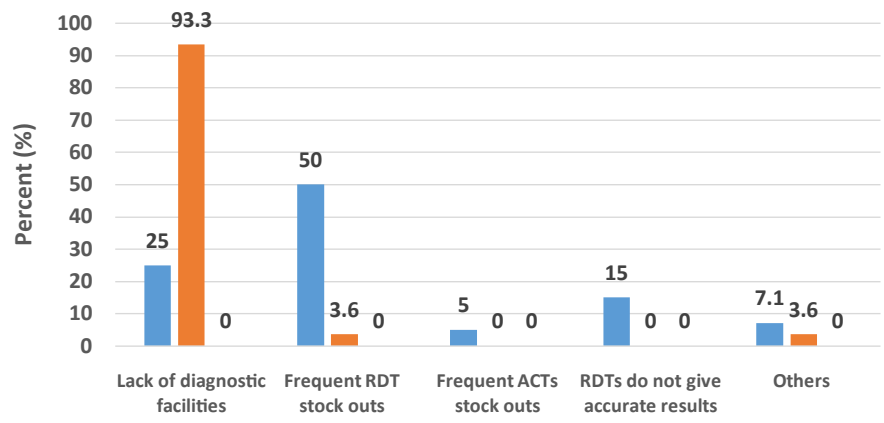

Figure 3. Challenges to the implementation of $\mathrm{T} 3$ strategy at various health facility levels.

[7]. This study revealed that the overall proportion of malaria cases tested was high (91.2\%). The District Hospital tested 97.5\% of its cases followed by the health centres, $89.5 \%$ and the CHPS compounds, $89.1 \%$. Although our findings could not reach the $100 \%$ target set by WHO, this study has found very impressive testing results compared to $38 \%$ reported by Nyandigisi in Kenya [11] and $27 \%$ by Osei-Kwakye 
in Ghana [12]. A study by Kankpetinge (Unpublished data) in the Ho municipality of Ghana in 2015 found a testing rate of $58.5 \%$. This could be due to the fact that Ho is an urban setting with private health facilities which may not be interested in testing free of charge and also write reports to the health directorate who supplied the RDTs.

Majority, (98.8\%) of the children tested for malaria were positive in this study. This is contrary to $52.8 \%$ positive rate found by Kankpetinge (Unpublished Data). The test rate among children reporting with fever was $92.7 \%$. Testing fever cases was higher (98.7\%) at the District Hospital level than at the CHPS compounds, (91.4\%) and Health Centres (90.5\%). This is because the District Hospital has both microscopy and RDTs for testing whilst the Health Centres and CHPS compounds lack microscopy technique so they are unable to test fever cases when they experienced RDTs stock-outs.

This study found that all the 353 children enrolled in the study were treated for malaria. Almost all (99.4\%) were treated with ACTs and only $0.6 \%$ were treated with Quinine which is for severe malaria or used as a second line drug for those not responding to ACTs. Majority, (91.2\%) were treated with ACTs base on test results, while $8.8 \%$ were treated with ACTs without any test. Thus, outcome of the tests did not in any way influenced treatment. This is similar to what was found in the Ho municipality (Kankpetinge, unpublished data).

According to Thiam et al. [17] the ability to track malaria cases and the impact of antimalarial interventions through the T3 strategy will enable malaria elimination. It is therefore expected that all treated cases would have returned for review in order to ascertain their cure status.

This study found overall, tracking of treated cases, which involved asking patients to return for review after receiving treatment, was (47.3\%). Only $21.8 \%$ were asked to return on specific days while $25.5 \%$ were asked to return only if the child's condition did not improve. CHPS compounds were more likely to ask patients to return for review, (70.6\%) compared to the hospital, (39.5\%) and the Health Centres (33.3\%). This could be due to the fact that CHPS compounds are closer to the people both socially and by distance.

It was also found that Doctors were involved in tracking malaria cases than other practitioners in the health facilities. Even though the numbers attended to, were few, 4 out of the 5 fever cases seen by the Doctor were asked to return for review as compared to the nurse (55.9\%), and Health Assistant, (51.1\%). Our study found that Medical/ Physician Assistants, (28.3\%) performed poorly in asking patients to return for review.

Using cases being asked to return for review as a proxy for tracking, compliance with the T3 (tested, treated and track) policy was estimated at $42.5 \%$. Majority (57.5\%) of the children missed at least a $\mathrm{T}$ and thus did not complete the protocol. The T3 is a combination of testing, treating and tracking. The Performance of these variables was therefore fed into compliance with T3. A bivariate correlation revealed that a case being asked to return for review $\left(\mathrm{R}^{2}=0.91\right.$ at $\left.\mathrm{p}<0.001\right)$ was the key determinant of completion of T3 compared to Testing $\left(\mathrm{R}^{2}=0.27\right.$ at $\mathrm{p}<0.001)$ and Treatment $\left(\mathrm{R}^{2}=0.07\right.$ at $\left.\mathrm{p}=0.224\right)$.

Compliance at the CHPS compounds was higher, (60.5\%) compared to that of the hospital, (39.5\%) and Health Centres, (30.1\%). It was observed that compliance followed the same pattern as that of the review. Therefore, the CHPS compounds did very well in complying with the T3 strategy as compared to the other levels as a result of asking children to return for review.
Doctors also complied with the $\mathrm{T} 3$ more than any other category of clinicians (4 out of 5), and this was as a result of asking cases to return for review compared to Assistant/Enrolled Nurses, (51.1\%), Nurses, (47.2\%) and Medical/Physician assistants (28.3\%). Although performance of the Doctor was very good, it did not reflect in the performance of the District Hospital. This could be because the Doctor attended to very few cases while majority of the cases were attended to by the MA/PA.

According to the clinicians, some of the challenges to T3 implementation were frequent RDT stock-outs, (39.2\%), lack of diagnostic facilities, (35.7\%) and frequent ACTs stock-outs (3.6\%). The major challenge at the CHPS compounds was frequent RDT stock-outs. The CHPS compounds were most affected because RDT was the only diagnostic tool available for testing. Health Centres also mentioned lack of diagnostic facilities such as microscopy as a major challenge. It was not surprising that the hospital did not have any challenge since both microscopy and RDTs were available [24-26].

\section{Limitations}

This study was a cross-sectional survey and could not capture time (season of the year and time of the day) element, which could have added more revealing reasons and recommendations. Recall issue with respondents even though was minimized by using exit interview, could have influenced their responses especially with their children being sick.

\section{Conclusion}

Compliance to the T3 was poor. However, it was encouraging to observe that malaria test rate in the Bongo district was very high and ACT treatment was the main drug of choice. Testing could not be done based on RDTs stock-out. The main determinant for the T3 was asking clients to return for clinical review. Health facility level such as CHPS compounds and clinicians such as Doctors and health assistants were more likely to comply with the T3 strategy.

\section{Recommendations}

NMCP should ensure that CHPS compounds and Health Centres are regularly stocked with RDTs and ACTs more than higher levels. In order to ensure compliance with T3 strategy, MA/PA and nurses would require training, monitoring and supervision on tracking of patients. Surveillance would improve if Health Centres are provided with microscopy services in addition to RDTs, which could also be used to provide other services apart from malaria diagnosis. There should be regular supply of ACTs to CHPS compounds and Health Centres.

\section{Declarations}

\section{Ethics and consent statement}

Ethical clearance was obtained from the Ghana Health Service Ethical Review Committee (GHS-ERC) with the approval identity (ID NO: GHS-ERC-12/02/16). Permission was also sought from the municipal health directorate and the heads of the health facilities. Moreover, the parents/guardians of the children consented to be part of the study.

\section{Availability of data and material}

Available upon request.

\section{Competing interests}

The authors declare that they have no competing interests 
Aganda S, et al. (2016) Implementation and challenges of test, treat and track (T3) strategy for malaria case management in children under five years in the Bongo District, Ghana

\section{Funding}

None

\section{Authors' contributions}

SN and MK conceived the study, MK, SN WT, EA, and ET did the data analysis and wrote the methods section. SN, MK, EA, MT and ET were responsible for the initial draft of the manuscript. All authors reviewed and approved the final version of the manuscript.

\section{Acknowledgements}

We are grateful to the staff of the School of Public Health Research Laboratory, University of Health and Allied Sciences. We are also grateful to the staff of the Bongo District Health Directorate. We would like to thank heads of the health facilities included in the study, the interviewers and the mothers/guardians of the children who participated in the study.

\section{References}

1. WHO (2009) World malaria report. World Health Organization, Geneva, Switzerland.

2. Nsimba SE, Massele AY, Eriksen J, Gustafsson LL, Tomson G, et al. (2002) Case management of malaria in under-fives at primary health care facilities in a Tanzanian district. Trop Med Int Health 7: 201-209. [Crossref]

3. Bisoffi Z, Sirima BS, Angheben A, Lodesani C, Gobbi F, et al. (2011) Strict adherence to malaria rapid test results might lead to a neglect of other dangerous diseases: a cost benefit analysis from Burkina Faso. Malaria Journal 10: 226

4. Abdelgader M, Ibrahim AM, Elmardi KA, Githinji S, Zurovac D, et al. (2011) Progress towards implementation of ACT in malaria case management in public health facilities in the Republic of Sudan, a cluster-sample survey. BMC Public Health 12: 11.

5. Bastiaens GJH, Bousema T, Leslie T (2014) Scale-up of Malaria Rapid Diagnostic Tests and Artemisinin-Based Combination Therapy: Challenges and Perspectives in Sub-Saharan Africa. PLoS Med 11: e1001590.

6. Crowell V, Yukich JO, Briet OJT, Ross A, Smith TA (2013) A Novel Approach for Measuring the Burden of Uncomplicated plasmodium falciparum Malaria: Application to Data from Zambia. Plos One 8: e57297.

7. Comoé CC, Ouattara AF, Raso G, Tanner M, Utzinger J, et al. (2012) Willingness to use a rapid diagnostic test for malaria in a rural area of central Côte d'Ivoire. $B M C$ Public Health 12: 1089. [Crossref]

8. Ayayi NA, Ukwaja KN (2013) Possible artemesinin-based combination therapyresistence malaria in Nigeria: a report of three cases. Revista da sociedade Brasileira de medicina Tropical 46: 525-527.
9. Udoh E, Oyo-ita A, Odey F, Effa E, Esu E, et al. (2013) Management of uncomplicated malaria in underfive in private and public health facilities in South-Eastern Nigeria: A clinical Audit of current practices. Hindawi Publishing Corporation.

10. Baiden F, Malm K, Bart-plange C, Hodgson A, Chandramohan D, et al. (2014) Shifting from presumptive to test-based management of malaria-technical basis and implications for malaria control in Ghana. Ghana Med J 48: 112-122. [Crossref]

11. Nyandigisi A, Memusi D, Mbithi A, Ang'wa N, Shieshia M, et al. (2011) Malaria case-management following change of policy to universal parasitological diagnosis and targeted artemisinin-based combination therapy in Kenya. PLOS ONE 6: e24781.

12. Osei-Kwakye K1, Asante KP, Mahama E, Apanga S, Owusu R, et al. (2013) The benefits or otherwise of managing malaria cases with or without laboratory diagnosis: the experience in a district hospital in Ghana. PLoS One 8: e58107. [Crossref]

13. WHO (2013) World malaria report. World Health Organization, Geneva, Switzerland.

14. Thiam S, Thior M, Faye B, Ndiop M, Diouf ML, et al. (2011) Major reduction in antimalarial drug consumption in Senegal after nation-wide introduction of malaria rapid diagnostic tests. PLoS One 6: e18419. [Crossref]

15. WHO (2012) Test. Treat. Track: Scaling up diagnostic testing, treatment and surveillance for malaria. Global Malaria Program Test, World Health Organization, Geneva, Switzerland.

16. Ministry of Health (2014) Guidelines for case management of malaria in Ghana. (3 $3^{\text {rd }}$ Edn), Ghana.

17. Global Malaria Program (2009) Malaria case management: Operations manual. World Health Organization, Geneva.

18. Kwarteng A, Asante KP, Abokyi L, Gyaase S, Febir LG, et al. (2015) Provider compliance to artemisinin-based combination therapy at primary health care facilities in the middle belt of Ghana. Malaria Journal 14: 361

19. Bongo District Health Directorate (2014) District Health Information Managemen systems DHIMS II: The Data Challenge for Ghana Health Service. Nethope.

20. GSS (2010) Ghana statistical services. Statistical service.

21. WHO (2010) Guidelines for the treatment of malaria. ( $2^{\text {nd }}$ Edn) World Health Organization Geneva.

22. WHO (2010) World malaria report. World Health Organization, Geneva, Switzerland.

23. WHO (2012) Disease surveillance for malaria elimination: operational manual. World Health Organization.

24. Basommi LB (2011) Spatial analysis of malaria epidemiology in the Amanse West District. Thesis submitted to the Department of Geomatic Engineering, Kwame Nkrumah University of Science and Technology, Ghana.

25. GHS (2014) National malaria control program, Ghana Health Service 2014 Annual Report.

26. Marta Jozef Majoros (2011) Treatment of uncomplicated malaria in Adults at Dormaa Presbyterian hospital, Dormaa Ahenkro. Manakin 18: 59Z.

Copyright: (C2016 Aganda S. This is an open-access article distributed under the terms of the Creative Commons Attribution License, which permits unrestricted use, distribution, and reproduction in any medium, provided the original author and source are credited. 